Prepared in cooperation with Bureau of Land Management and the National Renewable Energy Laboratory

\title{
GenEst Statistical Models-A Generalized Estimator of Mortality
}

Chapter 2 of

Section A, Algorithm

Book 7, Automated Data Processing and Computations

Techniques and Methods 7-A2 



\section{GenEst Statistical Models-A Generalized Estimator of Mortality}

By Daniel Dalthorp, Lisa Madsen, Manuela Huso, Paul Rabie, Robert Wolpert, Jared Studyvin, Juniper Simonis, and Jeffrey Mintz

Chapter 2 of

Section A, Algorithms

Book 7, Automated Data Processing and Computations

Prepared in cooperation with Bureau of Land Management and the National Renewable Energy Laboratory

Techniques and Methods 7-A2

U.S. Department of the Interior

U.S. Geological Survey 


\section{U.S. Department of the Interior \\ RYAN K. ZINKE, Secretary}

\section{U.S. Geological Survey James F. Reilly II, Director}

U.S. Geological Survey, Reston, Virginia: 2018

For more information on the USGS—-the Federal source for science about the Earth, its natural and living resources, natural hazards, and the environment-visit https://www.usgs.gov/ or call 1-888-ASK-USGS (1-888-275-8747).

For an overview of USGS information products, including maps, imagery, and publications, visit https:/store.usgs.gov.

Any use of trade, firm, or product names is for descriptive purposes only and does not imply endorsement by the U.S. Government.

Although this information product, for the most part, is in the public domain, it also may contain copyrighted materials as noted in the text. Permission to reproduce copyrighted items must be secured from the copyright owner.

Suggested citation:

Dalthorp, D., Madsen, L., Huso, M., Rabie, P., Wolpert, R., Studyvin, J., Simonis, J., and Mintz, J., 2018, GenEst statistical models-A generalized estimator of mortality: U.S. Geological Survey Techniques and Methods, book 7 , chap. A2, 13 p., https://doi.org/10.3133/tm7A2.

ISSN 2328-7055 (online) 


\section{Contents}

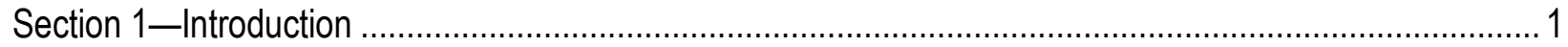

Section 2-Splitting Mortality Estimates by Carcass and Recombining into Subgroups............................. 3

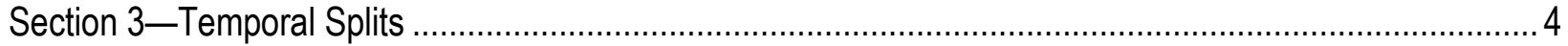

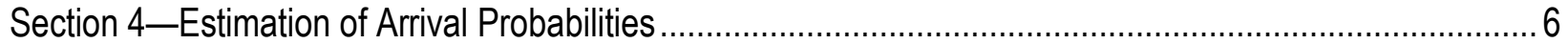

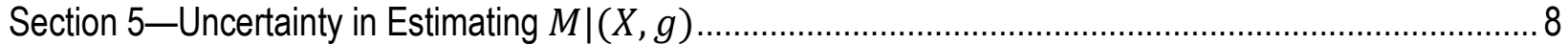

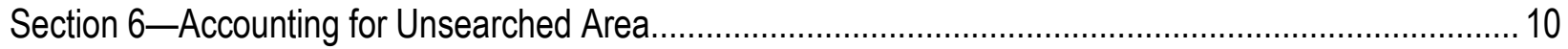

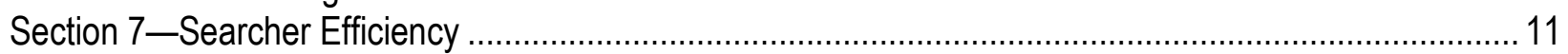

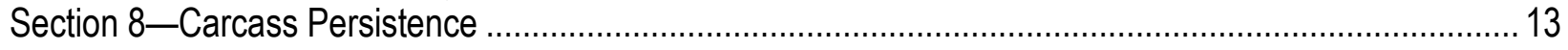

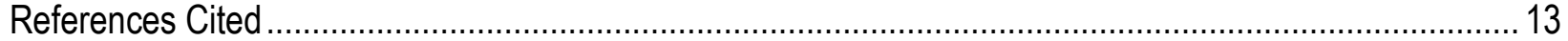

\section{Abbreviations}

$\begin{array}{ll}\text { A } & X \times n \text { sim array of simulated arrival intervals } \\ A_{j} & \text { event that the carcass arrived in interval } j \text { (between times } t_{j-1} \text { and } t_{j} \text { ) } \\ \text { CDF } & \text { cumulative distribution function } \\ \text { CP } & \text { carcass persistence } \\ d w p_{u} & \text { density-weighted proportion or the fraction of carcasses arriving within the searched area at search unit } u \\ f & \text { sampling fraction or the fraction of carcasses at a site that fall at the units selected for search } \\ \text { EoA } & \text { Evidence of Absence software and statistical model } \\ g, \hat{g} & \text { carcass detection probability, estimated carcass detection probability for carcasses arriving in searched areas } \\ \text { GenEst } & \text { Generalized Estimator of Mortality software and statistical model } \\ I_{j} & t_{j}-t_{j-1}=\text { length of search interval } j \\ k & \text { fractional change in searcher efficiency with each successive search after carcass arrival } \\ M, \widehat{M} & \text { mortality, estimated mortality } \\ n s e a r c h & \text { number of carcass search days during the monitored period } \\ n s i m & \text { number of simulation replicates for accounting for variance } \\ O_{i} & \text { event that the carcass is observed in search } i \text { (at time } t_{i} \text { ) } \\ p & \text { searcher efficiency for carcasses in the first search after carcass arrival } \\ \text { SE } & \text { searcher efficiency } \\ S(t) & \text { carcass persistence distribution giving the probability a carcass persists } t \text { or more days after carcass arrival } \\ t_{j} & \text { time (in days) of the } j \text { th search after the start of monitoring } \\ X & \text { number of carcasses observed }\end{array}$


This page left intentionally blank 


\section{GenEst Statistical Models-A Generalized Estimator of Mortality}

By Daniel Dalthorp', Lisa Madsen², Manuela Huso1, Paul Rabie³, Robert Wolpert4, Jared Studyvin³, Juniper Simonis ${ }^{5}$, and Jeffrey Mintz ${ }^{1}$

\section{Section 1-Introduction}

GenEst (a generalized estimator of mortality) is a suite of statistical models and software tools for generalized mortality estimation. It was specifically designed for estimating the number of bird and bat fatalities at solar and wind power facilities, but both the software (Dalthorp and others, 2018) and the underlying statistical models are general enough to be useful in various situations to estimate the size of open populations when detection probabilities and search coverages are less than 1 . In this report, we outline the statistical models and data structures underlying the estimator. The models are numerous, complex, and intricately interwoven. Discussion begins with broad, high-level overviews of the general models. The lower-level technical details are then gradually added. Broader and less technical discussions on the general context and applications of the models and the use of the software are available in the software user guide (Simonis and others, 2018), vignettes bundled with the software, and the help files within the software itself.

\footnotetext{
${ }^{1}$ U.S. Geological Survey.

${ }^{2}$ Oregon State University.

${ }^{3}$ Western EcoSystems Technology, Inc.

${ }^{4}$ Duke University

${ }^{5}$ DAPPER Stats.
} 
At its core, GenEst is an elaboration of a binomial probability model $X \sim \operatorname{binomial}(M, g)$, where $X$ is the observed number of carcasses and $g$ is the detection probability. If $g$ is known, then $\widehat{M}=$ $X / g$ is an unbiased estimator for $M$ and the sampling variance of $X$ is the only source of uncertainty about $M$. In a slightly more complicated scenario in which total mortality is split into two groups, $A$ and $B$, then $\widehat{M}=\widehat{M}_{A}+\widehat{M}_{B}=\frac{X_{A}}{g_{A}}+\frac{X_{B}}{g_{B}}$ is unbiased for $M$. GenEst makes extensive use of this simple idea of splitting the carcass observation data into distinct subgroups, estimating mortality in each subgroup, and combining subgroups into larger groups by summing. A number of technical difficulties must be overcome to make this simple idea work as a complete estimator that produces accurate confidence intervals, including accounting for the dependence of $g$ on the time of carcass arrival, estimating $g$ as a function of covariates, characterizing the uncertainty in $\hat{g}$, accounting for correlation structure of the $\hat{g}^{\prime}$ s among various subgroups, accounting for uncertainty in estimating $M$ given $X$ and $g$, and accounting for unsearched area. GenEst provides solutions for each of these difficulties. The technical details are lengthy but are fully explained in sections $2-8$. 


\section{Section 2-Splitting Mortality Estimates by Carcass and Recombining into Subgroups}

For each carcass, $x=1, \ldots, X$, that is discovered in carcass surveys, its contribution to the estimated total mortality is the reciprocal of its inclusion probability, $1 / g_{x}$. However, that contribution is subject to two types of uncertainty: (1) uncertainty associated with estimating $g_{x}$ (sections 7-8), and (2) the uncertainty associated with estimating $M_{x} \mid g_{x}$ (section 5). We account for the uncertainties using parametric bootstrapping, that is, simulating from the asymptotic distributions of the maximum likelihood estimators of parameters. The result is stored in an $X \times n \operatorname{sim}$ matrix of carcass contributions to the estimated total mortality:

$$
\widehat{\mathbf{M}}=\left[\begin{array}{cccc}
\widehat{m}_{1,1} & \widehat{m}_{1,2} & \cdots & \widehat{m}_{1, n s i m} \\
\widehat{m}_{2,1} & \widehat{m}_{2,1} & \cdots & \widehat{m}_{2, n s i m} \\
\vdots & \vdots & \ddots & \vdots \\
\widehat{m}_{X, 1} & \widehat{m}_{X, 2} & \cdots & \widehat{m}_{X, n s i m}
\end{array}\right]
$$

Each column of $\widehat{\mathbf{M}}$ represents a single realization of the simulated contributions of all the discovered carcasses to the estimated total mortality. Uncertainty is captured in the distinctions among columns.

Mortality estimation by carcass group is accomplished by subsetting the rows and then taking column sums. For example, total mortality (no subsetting) is estimated by taking column sums over all the carcasses to create a vector of estimates, $\widehat{M}_{\text {total }}=\left(\sum_{x} \widehat{m}_{x, 1}, \sum_{x} \widehat{m}_{x, 2}, \ldots, \sum_{x} \widehat{m}_{x, n s i m}\right)$, from a parametric bootstrap from the sampling distribution of $\widehat{M}$. The $\widehat{\mathbf{M}}$ matrix can be subsetted to estimate mortality among desired subgroups in a similar way. For example, to estimate mortality for species B, calculate $\widehat{M}_{\mathrm{B}}=\left(\sum_{x \in \mathrm{B}} \widehat{m}_{x, 1}, \sum_{x \in \mathrm{B}} \widehat{m}_{x, 2}, \ldots, \sum_{x \in \mathrm{B}} \widehat{m}_{x, n s i m}\right)$, where $x \in \mathrm{B}$ indicates that carcass $x$ belongs to species B. GenEst extracts sample statistics (such as median and confidence intervals) from these empirical $\widehat{M}$ vectors to summarize mortality estimates for subgroups or "splits" as defined by the user. 


\section{Section 3-Temporal Splits}

GenEst can also split mortality estimates by user-specified time intervals or by temporal covariates such as season. To split the $\widehat{\mathbf{M}}$ matrix by carcass arrival times, GenEst relies on an $X \times n s i m$ matrix of simulated arrival intervals, $\mathbf{A}$, to construct an ntimes $\times$ nsim array of mortality estimates by time interval, $\widehat{M}_{T}$, as:

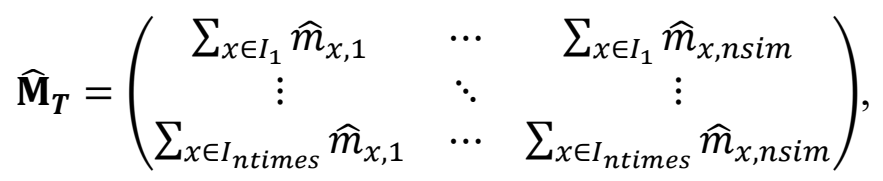

where $x \in I_{j}$ indicates that the simulated arrival time of carcass $x$ is within the specified interval, $j$. If the simulated arrival interval of a carcass $x_{i}$ intersects two or more of the splitting intervals (for example, $I_{j}$ and $\left.I_{j-1}\right)$, the contribution of the carcass to estimated mortality, $\widehat{m}$, is allocated proportionally to the intersecting splitting intervals.

To understand the structure of the $\mathbf{A}$ matrix, first note that detection probability for a carcass depends partly on its arrival time. For example, if monitoring runs from April 1 through October 31, carcasses arriving near the end of October may be available for discovery for only one or two searches, whereas carcasses arriving in April may be available for many searches. Search conditions also may vary with season. However, although carcass discovery times are recorded, arrival times are not known. Discovered carcasses may have arrived sometime after the previous search or in any search interval prior to that. Although arrival times cannot be known with certainty, GenEst analyzes the search data to derive probability distributions of arrival times for each carcass and, from these, creates an $X \times n \operatorname{sim}$ matrix of simulated arrival intervals, A. For example, if $X=3$ and the carcasses were discovered on searches $i=1,3$, and 12 , respectively, and $n \operatorname{sim}=10$, the arrival matrix, $\mathbf{A}$, might look like the following:

$$
\mathbf{A}=\left[\begin{array}{cccccccccc}
1 & 1 & 1 & 1 & 1 & 1 & 1 & 1 & 1 & 1 \\
3 & 3 & 2 & 3 & 1 & 3 & 3 & 3 & 2 & 1 \\
12 & 11 & 12 & 10 & 9 & 12 & 12 & 12 & 12 & 11
\end{array}\right]
$$


The simulated arrival intervals for the first carcass are identically 1 because the carcass was discovered on the first search (at $\left.t_{1}\right)$ and is assumed to have arrived in the first search interval, $\left(t_{0}, t_{1}\right]$. In practice, the assumption that all carcasses discovered in carcass surveys arrived after $t_{0}$ is often enforced by disregarding carcasses found in a careful clean-out search at $t_{0}$. The second carcass was discovered in search 3. According to the arrival probabilities, the carcass was more likely to have arrived in interval 3 than in any other interval, but there is a chance it arrived as early as the first interval, as indicated by the row of simulated arrivals. The third carcass was found on the 12th search and, in theory, could have arrived in any interval prior to its discovery. However, it is highly unlikely that it arrived more than a few intervals prior to the 12 th search because, if it had, chances are that it would have been removed by scavengers or previously found by searchers. 


\section{Section 4-Estimation of Arrival Probabilities}

Suppose searches are conducted at times, $t_{0}, t_{1}, \ldots, t_{n s e a r c h}$. For each carcass discovered in carcass surveys $i=1, \ldots$, nsearch, define $O_{i}$ as the event that the carcass was observed during search $i$ (at time $t_{i}$ ) and $A_{j}$ as the event that the carcass arrived in interval $j=\left(t_{j-1}, t_{j}\right], j \leq i$. The probability that the carcass arrived in interval $j$ then can be estimated as:

$$
\operatorname{Pr}\left(A_{j} \mid O_{i}\right)=\frac{\operatorname{Pr}\left(O_{i} \mid A_{j}\right) \operatorname{Pr}\left(A_{j}\right)}{\sum_{j \leq i} \operatorname{Pr}\left(O_{i} \mid A_{j}\right) \operatorname{Pr}\left(A_{j}\right)}
$$

In theory, $\operatorname{Pr}\left(A_{j} \mid O_{i}\right)$ is positive for $j=i, i-1, \ldots, 1$, but, in practice, $\operatorname{Pr}\left(A_{j} \mid O_{i}\right)$ decreases rapidly to 0 with $j$, so in most cases only the first few terms need to be calculated. The default for GenEst software is to calculate arrival probabilities for as many as eight intervals prior to discovery, but the R commandline user may override the default in functions estM and estg. GenEst makes a further assumption that the arrival rate is constant in the search intervals preceding carcass arrival, so

$$
\operatorname{Pr}\left(A_{j} \mid O_{i}\right)=\frac{\operatorname{Pr}\left(O_{i} \mid A_{j}\right) / I_{j}}{\sum_{j \leq i} \operatorname{Pr}\left(O_{i} \mid A_{j}\right) \operatorname{Pr}\left(A_{j}\right) / I_{j}}
$$

where $I_{j}=t_{j}-t_{j-1}=$ length of interval $j$.

The estimator is robust to variation in arrival rates among different search intervals provided there is not an abrupt change in arrival rate from one interval to the next. However, even in these unusual conditions, the potential for bias in estimating total mortality would be small because the same pattern would need to recur with a large fraction of the carcasses. The potential for bias would be further attenuated by high searcher efficiency [SE] or low persistence times. 
Calculation of $\operatorname{Pr}\left(A_{j} \mid O_{i}\right)$ is based on calculation of $\operatorname{Pr}\left(O_{i} \mid A_{j}\right)$ :

$$
\operatorname{Pr}\left(O_{i} \mid A_{j}\right)= \begin{cases}0, & i<j \\ p \int_{t_{j-1}}^{t_{j}} \frac{S\left(t_{i}-t\right)}{I_{j}} d t, & i=j \\ {\left[\prod_{s=0}^{i-j-1}\left(1-p k^{s}\right)\right] p k^{i-j} \int_{t_{j-1}}^{t_{j}} \frac{S\left(t_{i}-t\right)}{I_{j}} d t,} & i>j\end{cases}
$$

where $S(t)$ is the probability that a carcass persists $t$ days after arrival (section 8), $p$ and $k$ are searcher efficiency parameters (section 7), and $s$ (the index on the product) runs from the search interval immediately before carcass discovery to the first search interval of the monitored period. A carcass may arrive in one season and be discovered in a later season after search conditions have changed. To account for this possibility, in the calculation of $\operatorname{Pr}\left(O_{i} \mid A_{j}\right)$ of a carcass for a given $j$, the carcass is assigned the search characteristics appropriate to the assumed arrival interval, $j$. For example, if a carcass is discovered on the first search in the autumn, and search conditions (that is, SE and carcass persistence $[\mathrm{CP}]$ parameters) change from summer to autumn, then $\operatorname{Pr}\left(O_{i} \mid A_{i}\right)$ is calculated with the autumn SE and CP parameters and $\operatorname{Pr}\left(O_{i} \mid A_{i-1}\right)$ is calculated with the summer parameters.

Arrival-interval probabilities, $\operatorname{Pr}\left(A_{j} \mid O_{i}\right)$, are calculated for each carcass and search interval, and an integer-valued $X \times n \operatorname{sim}$ matrix of simulated arrival intervals, $\mathbf{A}$, is constructed. Each column represents the arrival interval of each carcass in a simulated set of carcass arrivals. In other words, each column represents simulated arrival intervals of the set of observed carcasses over the course of one field season or simulated "year". As mentioned previously, carcass detection probabilities depend on arrival times, which are given in the arrival matrix $\mathbf{A}$. For each simulated year (column in $\mathbf{A}$ ), the detection probability for each carcass is estimated as

$$
\hat{g}=\operatorname{Pr}\left(O \mid A_{j}\right)=\sum_{i} \operatorname{Pr}\left(O_{i} \mid A_{j}\right)
$$

to construct an $X \times n \operatorname{sim}$ matrix, $\hat{g}$, of carcass detection probabilities that are applicable to the carcass at the time of carcass arrival. For example, $\hat{g}_{x, j}$ is a simulated inclusion probability of carcass $x$, assuming it arrived in interval $A_{x, j}$. 


\section{Section 5-Uncertainty in Estimating $M \mid(X, g)$}

The uncertainty in estimating $g_{x}$ for carcass $x$ is captured in $\hat{\mathbf{g}}_{x, \cdot}=\left(\hat{g}_{x, 1}, \ldots, \hat{g}_{x, n s i m}\right)$, but there remains much uncertainty about the true mortality, $M$, even if $g$ is known, owing to the sampling variation in $X$ or the uncertainty in estimating $M$. GenEst accounts for that uncertainty in a novel way that lends itself well to the framework of splits. The process is to estimate the sampling variation in $X$, rescale the estimated variation to $\widehat{M}$, and adjust for bias.

We can assume $X \sim \operatorname{binomial}(M, g)$, but, unfortunately, we do not know $M$. We can, however, estimate $M$ by $X / g$ and (almost) define a new random variable $\tilde{X} \sim \operatorname{binomial}(X / g, g)$ to account for the variation in $X \mid(M, g)$. The uncertainty in estimating $M$ would then be accounted for in $\widehat{M}=\tilde{X} / g$. However, $X / g$ might not be an integer and cannot serve as the index for a binomial random variable. Instead, we define $\tilde{X} \sim \operatorname{cbinom}(X / g, g)$, where cbinom is a continuous generalization of the standard binomial distribution (Ilienko, 2013), which is implemented in the R package cbinom (Dalthorp, 2018). 
The continuous binomial spreads the probability mass of the binomial distribution on each integer $k$ to the interval $[k, k+1)$, so the distribution is a smoothed version of the binomial but shifted slightly to the right. The mean of the continuous binomial distribution with parameters $m$ and $g$ is $\int_{0}^{m+1}[1-F(x ; m, g)] d x$, where $F$ is the CDF of the continuous binomial:

$$
F(x ; m, g)=\left\{\begin{array}{lr}
0, & x \leq 0 \\
B(x, m+1-x, g) & 0<x \leq m+1 \\
B(x, m+1-x, 0) & x>m+1
\end{array}\right.
$$

and

$$
B(x, y, g)=\int_{g}^{1} t^{x-1}(1-t)^{y-1} d t
$$

is the incomplete beta function. The expectation of a random variable $\tilde{X} \sim \operatorname{cbinom}(m, g)$ is

$$
\mathrm{E}[\tilde{X}]=\int_{0}^{m+1}[1-F(x ; m, g)] d x
$$

which is approximately $m g+0.5$ and can be calculated numerically. Because the expected value of a binomial random variable $X \sim \operatorname{binomial}(m, g)$ is $m g, \tilde{X}$ exceeds $X$ by about 0.5 . Thus, $\tilde{X} / g$ would be biased for estimating $M$, so we subtract the bias before dividing by $g$ :

$$
\widehat{M}=\frac{[\tilde{X}-(\mathrm{E}[\tilde{X}]-x)]}{g} .
$$

Because this estimator is unbiased, mortality estimates can be summed. For example, if $\widehat{M}_{A}$ is the estimated mortality in area $\mathrm{A}$ and $\widehat{M}_{B}$ is the estimated mortality in area $\mathrm{B}$, then $\widehat{M}_{\text {total }}=\widehat{M}_{A}+\widehat{M}_{B}$ is an unbiased estimator for the two areas combined. There is nothing special about "area" here; A and B could represent times, species, carcass sizes, search teams, turbine types, or other variables. GenEst takes this idea to the limit and builds the $X \times n \operatorname{sim}$ matrix, $\widehat{\mathbf{M}}$, in which each carcass represents its own "area". Users then define how they wish to split the overall mortality into summary groups. In the simplest case, total mortality is estimated as the sum of the contribution of each carcass to the total; that is, the column sums of the $\widehat{\mathbf{M}}$ matrix. 


\section{Section 6-Accounting for Unsearched Area}

Unless the user is interested in estimating mortality strictly in the searched area, $\hat{g}$ values must be adjusted to account for the unsearched area. In practice, some carcasses are likely to fall outside the searched area in a given unit (for example, in an unsearched part of a search ring at a solar power tower facility or beyond the search radius at a wind turbine). For each search unit, $u$, the expected fraction of carcasses that are killed at the unit but fall outside the searched area is the density-weighted proportion or $d w p_{u}$ (Huso and Dalthorp, 2014). Additionally, there may be units at a site that are not searched at all. The expected fraction of carcasses arriving at the units searched at a site is the sampling fraction or $f$. For example, if units at a wind facility are the individual turbines, then $f$ would be the fraction of turbines surveyed. Thus, the contributions of the respective carcasses to the estimate of total mortality are then summarized in a $X \times n \operatorname{sim}$ array as $\frac{1}{\mathbf{d w p} \cdot \hat{\mathbf{g}} \cdot f}$, where $\mathbf{d w p}=\operatorname{diag}(\mathbf{d w p})$ is the diagonal matrix of $d w p$ values associated with the carcassess. For example, if carcass 1 was found at unit 17 and was a medium-sized bird, then $d w p_{1,1}$ would be the $d w p$ for medium-sized birds at unit 17. $\widehat{\mathbf{M}}$ would then be calculated based on this adjusted $\hat{\mathbf{g}}_{*}=\mathbf{d w} \mathbf{p} \cdot \hat{\mathbf{g}} \cdot f$. 


\section{Section 7-Searcher Efficiency}

Searcher efficiency and carcass persistence (section 8) are estimated from field trials where a known number of carcasses are placed and their fate (found or scavenged) is recorded. Let $p$ be the initial SE for fresh carcasses, or, more precisely, the conditional probability of detecting a carcass on the first search after carcass arrival, given that the carcass is present at the time of the search. Let $k$ be the fractional change in SE with each successive search. Thus, if SE trial carcasses that are missed in one search are left in the field for possible discovery on later searches, $p$ and $k$ can be estimated simultaneously as functions of categorical covariates.

Specifically, let $p_{i}$ be the probability that carcass $i$ is found during the first search after carcass arrival given that it is present at the time of the search. The model allows $p_{i}$ to depend on a vector of covariates $\mathbf{X}_{i}$ as $\operatorname{logit}\left(p_{i}\right)=\mathbf{X}_{i} \beta$, where $\beta$ is a vector of coefficients associated with combinations of covariate levels. The model allows a constant multiplicative reduction in detection probability in subsequent searches. The probability of finding carcass $i$ during search $j$ is

$$
\operatorname{Pr}\{\text { detect carcass } i \text { on occasion } j\}=p_{i} k_{i}^{j-1}
$$

where $k_{i}$ may also be a logit-linear function of covariates and coefficients. These covariates need not be the same as those used to model $p_{i}$.

Consider the search outcome history for a given carcass. If the carcass was scavenged before the first search occasion, then the carcass provides no information to estimate SE, and the model ignores these carcasses. If the carcass is present during one or more search occasions, then the probability of its search history is determined by that history and equation. These relations are easiest to see with a couple of examples. Suppose carcass $i$ (from the SE field trials) was missed on three searches and detected on the fourth search. Its search history is then $(0,0,0,1)$ in the notation of the data, and the probability of this search history is

$$
\operatorname{Pr}\{(0,0,0,1)\}=\left(1-p_{i}\right)\left(1-p_{i} k_{i}\right)\left(1-p_{i} k_{i}^{2}\right) p_{i} k_{i}^{3}=L_{i}
$$

where $L_{i}$ denotes the contribution of carcass $i$ to the joint likelihood. 
A carcass may never be found. For example, suppose carcass $i^{\prime}$ was missed on the first two search occasions and was then scavenged before the third search. This carcass has search history $(0,0)$, and

$$
L_{i^{\prime}}=\left(1-p_{i^{\prime}}\right)\left(1-p_{i^{\prime}} k_{i^{\prime}}\right)
$$

The $\log$-likelihood of the data is then expressed as $\log L=\sum_{i=1}^{N} \log L_{i}$, where $N$ denotes the total number of carcasses in the trial. Let $z_{i}$ denote the number of zeros in the search history for carcass $i$ (that is, $z_{i}$ is the number of times carcass $i$ was missed in searches), and let $f_{i}$ denote the search occasion where the carcass was found, with $f_{i}=0$ if the carcass was never found. The, $\log L_{i}$ will then have the form

$$
\log L_{i}=\sum_{j=0}^{z_{i}-1} \log \left(1-p_{i} k_{i}^{j}\right)+\mathbf{1}_{f_{i}>0} \log \left(p_{i} k_{i}^{f_{i}-1}\right)
$$

where $\mathbf{1}_{f_{i}>0}=1$ if $f_{i}>0$ and zero otherwise. Thus, the log-likelihood depends on the data only through the number of missed searches and whether the carcass was eventually found.

The log-likelihood, $L$, of the data is numerically maximized in using R's optim function. From the theory of maximum likelihood, the vector of maximum likelihood estimators (MLEs) for the parameters $(\widehat{\beta})$ is asymptotically multivariate normal with mean equal to the true parameter values and variance-covariance matrix given by the inverse information matrix, which is returned by optim as the hessian.

To account for the uncertainty in estimating $p$ and $k$ parameters, GenEst first approximates the sampling distribution of $\widehat{\beta}$ by simulating from the multivariate normal (MVN),

$$
\widehat{\beta}_{\operatorname{sim}} \sim \operatorname{MVN}\left(\widehat{\beta}, \mathbf{H}^{-1}\right)
$$

Where $\mathbf{H}$ is the Hessian matrix returned by optim( ).

Simulated sampling distributions of $p_{i}$ and $k_{i}$ are obtained by back transformation of simulated $\hat{\beta}$. Specifically, let $\mathbf{X}_{i}$ represent the covariate vector for the $i$ th carcass, then

$$
\widehat{k}_{i}=\frac{1}{1+\exp \left(-\mathbf{X}_{i} \cdot \widehat{\beta}_{k}\right)} \text { and } \widehat{p}_{i}=\frac{1}{1+\exp \left(-\mathbf{X}_{i} \cdot \widehat{\beta}_{p}\right)}
$$

where $\hat{\beta}_{p}$ and $\hat{\beta}_{k}$ are the components of $\hat{\beta}$ associated with $p$ and $k$, respectively. 


\section{Section 8-Carcass Persistence}

Carcass persistence times are modeled using censored exponential, Weibull, lognormal, and loglogistic survival models, which are fit by maximum likelihood estimation using the R functions survival: : survreg (Therneau, 2015) and optim (R Core Team, 2018). Both the location and scale parameters (Kalbfleisch and Prentice, 2002, chapter 2) may depend on categorical covariates, such as visibility class, season, or other factors. As with the SE estimates, vectors of simulated persistence parameters are generated from the fitted models.

\section{References Cited}

Dalthorp, D., 2018, cbinom — Continuous analog of a binomial distribution-R package, version 1.3: Web page accessed October 19, 2018, at https://CRAN.R-project.org/package=cbinom.

Dalthorp, D., Simonis, J., Madsen, L., Huso, M., Rabie, P., Mintz, J., Wolpert, R., Studyvin, J., and Korner-Nievergelt, F., 2018, GenEst—Generalised fatality estimator-R package, version 1.0.0: Web page accessed October 19, 2018, at https://code.usgs.gov/ecosystems/GenEst/tags/1.0.0.

Huso, M.M.P., and Dalthorp, D., 2014, Accounting for unsearched areas in estimating wind turbinecaused fatality: The Journal of Wildlife Management, v. 78, no. 2, p. 347-358.

Ilienko, A., 2013, Continuous counterparts of Poisson and binomial distributions and their properties: Annales Universitatis Scientarium Budapestinensis, Sectio Computatorica, v. 39: p. 137-147.

Kalbfleisch, J.D., and Prentice, R.L., 2002, The statistical analysis of failure time data: Hoboken, New Jersey, Wiley, 462 p.

R Core Team, 2018, R-A language and environment for statistical computing: Vienna, Austria, R Foundation for Statistical Computing web page, accessed October 19, 2018, at https://www.Rproject.org/.

Simonis, J., Dalthorp, D., Huso, M., Mintz, J., Madsen, L., Rabie, P., and Studyvin, J., 2018, GenEst user guide: Software for a generalized estimator of mortality: U.S. Geological Survey Techniques and Methods, book 7, chap. C19, 72 p.

Therneau, T., 2015, Package for survival analysis in S, version 2.38: The Comprehensive R Archive Network web page, accessed October 19, 2018, at https://CRAN.R-project.org/package=survival. 

Publishing support provided by the U.S. Geological Survey Science Publishing Network, Tacoma Publishing Service Center

For more information concerning the research in this report, contact the Director, Forest and Rangeland Ecosystem Science Center U.S. Geological Survey

777 NW 9th St., Suite 400

Corvallis, Oregon 97330

https://www.usgs.gov/centers/fresc/ 


\section{寍}

임

음

움

9

寒

章

ㄹ.

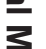

음

$>$

모ำ

\$ิ

蕓

罗

를

음

윽

를

$\frac{1}{10}$

올

品

ํㅡㄹ

3

웅

齐 